\title{
The front-end of IsoDAR
}

\section{Spencer N. Axani ${ }^{* \dagger}$}

The Massachusetts Institute of Technology

E-mail: saxani@mit.edu

\section{For the IsoDAR/DAE $\delta$ ALUS Collaboration}

The Isotope Decay-At-Rest (IsoDAR) experiment is a cyclotron based neutrino oscillation experiment that is capable of decisively searching for low-mass sterile neutrinos. This paper outlines two new approaches that the IsoDAR collaboration are pursuing in order to increase the amount of $\mathrm{H}_{2}^{+}$captured in the cyclotron through innovations in the design of the front-end. A new dedicated multicusp ion source (MIST-1) is currently being commissioned and tested at the Plasma Science and Fusion Center (PSFC) at MIT. Based on previous results from this type of ion source, we expect to be able to achieve an $\mathrm{H}_{2}^{+}$current density that will be sufficient for the IsoDAR experiment. We also discuss the results of a new investigation into using a radio frequency quadrupole (RFQ) as a high-efficiency buncher to improve the injection efficiency into the cyclotron.

38th International Conference on High Energy Physics

3-10 August 2016

Chicago, USA

\footnotetext{
* Speaker.

${ }^{\dagger}$ The authors would like to thank the NSF for their continued support. NSF Grant no. 1505858 and 1626069.
} 


\section{Introduction}

The Isotope Decay-At-Rest (IsoDAR) experiment is motivated by the set of recently observed anomalies in short baseline neutrino oscillation experiments. These anomalies are seen in electron neutrino disappearance $[1,2,3]$ and electron neutrino appearance $[4,5]$ oscillation channels. The anomalies can be resolved with the introduction of a new sterile neutrino state (one which does not interact via the weak nuclear force but is able to oscillate into the other active neutrino states). In a $3+1$ sterile neutrino model, where there are 3 active neutrino flavors and 1 sterile flavor, the oscillation amplitude associated with short baseline $v_{e}$ disappearance is proportional to $\left|U_{e 4}\right|^{2}(1-$ $\left.\left|U_{e 4}\right|^{2}\right)$, where $U_{e 4}$ is the mixing element from the extended PMNS matrix between the electron state and the sterile mass state. IsoDAR is an experiment that will make a precision measurement of the oscillation amplitude, and therefore the mixing element $U_{e 4}$, over a wide range of possible sterile neutrino masses. Further, IsoDAR can potentially distinguish between a $3+\mathrm{N}$ sterile neutrino model, and will collect the world's largest sample of $\bar{v}_{e}$ electron elastic scattering events [6].

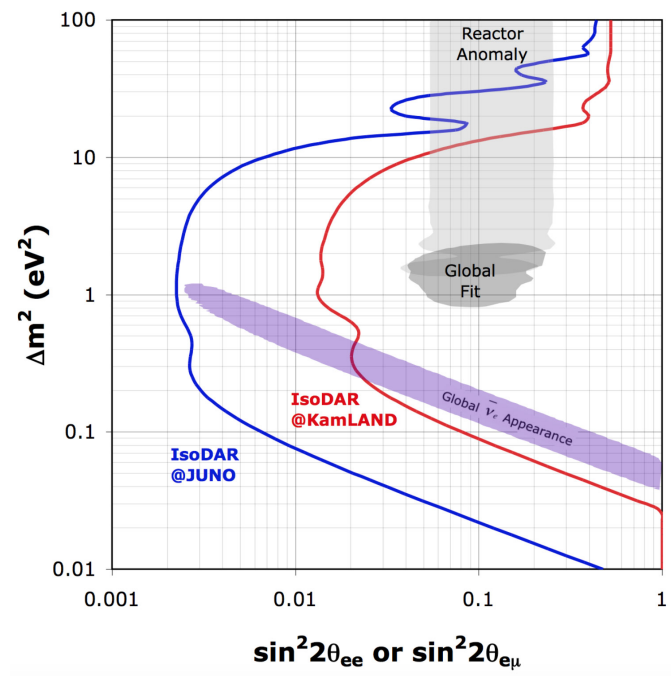

Figure 1: The projected $5 \sigma \mathrm{CL}$ of IsoDAR for a nominal 5-y run at KamLAND or JUNO. The light gray contour represents the $99 \% \mathrm{CL}$ allowed region for the reactor anomaly, from [9]; the dark gray represents the global allowed region, from [10]. This plot is reproduced from [11].

The IsoDAR neutrino source consists of a compact cyclotron that accelerates $\mathrm{H}_{2}^{+}$up to $60 \mathrm{MeV} / \mathrm{amu}$ and impinges the ions on a ${ }^{9} \mathrm{Be}$ target to produce a large flux of neutrons. The neutrons capture on a $>99.99 \%$ pure ${ }^{7} \mathrm{Li}$ cylindrical sleeve to produce ${ }^{8} \mathrm{Li}$. The ${ }^{8} \mathrm{Li}$ then undergoes a $\beta$-decays at rest, producing an isotropic $\bar{v}_{e}$ flux. Placing the IsoDAR neutrino source near a kiloton scale detector, like KamLAND [7] or JUNO [8], would enable a definitive sterile neutrino search by measuring the $\bar{v}_{e}$ disappearance throughout the detector. In five years of operation, IsoDAR paired with the KamLAND detector, could decisively rule out the entire global $3+1$ allowed region at $20 \sigma$ (see Fig. 1).

To achieve the desired neutrino flux, IsoDAR requires $10 \mathrm{~mA}$ of protons (or equivalently $5 \mathrm{~mA}$ of $\mathrm{H}_{2}^{+}$) to be extracted from the cyclotron. This is approximately 10 times the current performance of commercial cyclotrons at similar energies. Several innovations make this possible: IsoDAR will use $\mathrm{H}_{2}^{+}$, which reduces the space-charge forces on the beam and it will be able to inject a higher current into the cyclotron. In this paper, we present two new technologies that the collaboration is pursuing to improve the injected current into the cyclotron by modifying the front-end $\left(\mathrm{H}_{2}^{+}\right.$production, transport, and injection into the cyclotron) of IsoDAR . A new high-intensity $\mathrm{H}_{2}^{+}$multicusp ion source (MIST1) is currently being commissioned at the Plasma Science and Fusion Center (PSFC) at MIT, and a radio frequency quadrupole (RFQ) is being designed for use as a pre-accelerator, buncher, and focusing element prior to injection into the cyclotron. 


\section{MIST-1: the $\mathrm{H}_{2}^{+}$multicusp ion source for IsoDAR}

In 2014, the IsoDAR collaboration performed a series of measurements at Best Cyclotron Systems, Inc. in Vancouver, Canada, which were outlined in [12]. One of the key conclusions of the tests was that the extracted $\mathrm{H}_{2}^{+}$current from the Versatile Ion Source (VIS) [13] could be increased by decreasing the radial size of the plasma chamber and by increasing the area of the extraction aperture. Our simulations indicate that the VIS, with an increased extraction aperture, would provide the lower limit of the required current for IsoDAR. Since this limits the flexibility of the other components of the experiment, we are currently developing a new dedicated high-current $\mathrm{H}_{2}^{+}$multicusp ion source for IsoDAR, which we call MIST-1.
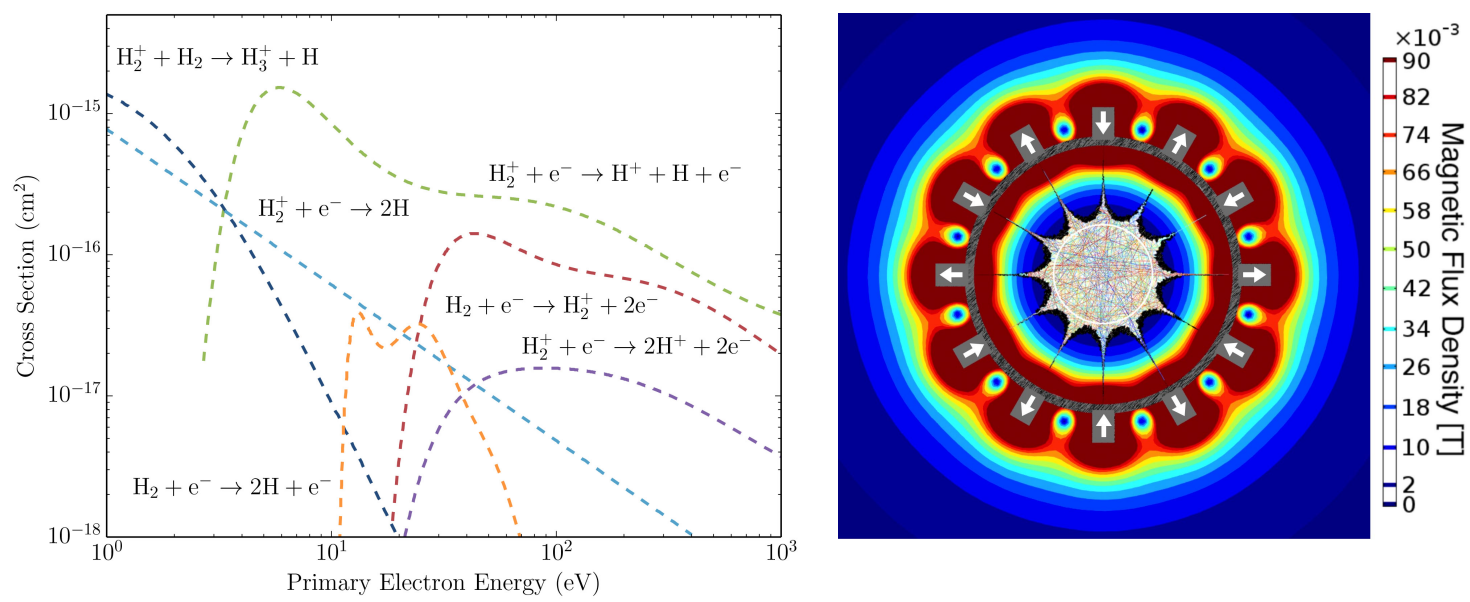

Figure 2: (Left) The cross-sections associated with the production and destruction processes of molecular hydrogen. The tabulated values were from the IAEA [14]. (Right) The simulated magnetic flux density near the extraction hole of the plasma chamber. The white arrows indicate the orientation of the $\mathrm{Sm}_{2} \mathrm{Co}_{17}$ magnets. Particle trajectories for 40 and $80 \mathrm{eV}$ electrons are shown. A description of the simulation results can be found in [15].

Several experimental measurements influenced the major design choices for MIST-1:

1. Based on the cross-sections, from the International Atomic Energy Agency (IAEA) [14], for the production and destruction processes of $\mathrm{H}_{2}^{+}$, the plasma volume must remain small so that the $\mathrm{H}_{2}^{+}$ions can be extracted before the destruction processes begin to dominate (see Fig. 2 (left)). We limit our plasma volume using $\mathrm{Sm}_{2} \mathrm{Co}_{17}$ magnets such that the plasma is contained within a volume smaller than the mean free path $\mathrm{H}_{2}^{+}$(a few centimeters).

2. The configuration of the permanent magnets that form the magnetic field must be placed in such a way to produce a quiescent, field free region near the extraction aperture. This is accomplished using a multicusp magnetic arrangement on the front plate, plasma chamber, and back plate (see Fig. 2 (right)).

3. Reference [16] concluded that biasing their plasma chamber with respect to the end flange by $+30 \mathrm{~V}$ increased the total current density of $30 \%$, with a $2 \%$ decrease in $\mathrm{H}_{2}^{+}$fraction. Our design will incorporate these results. 
4. $\mathrm{Sm}_{2} \mathrm{Co}_{17}$ rare earth magnets are preferred for generating the magnetic field due to their heat properties and high magnetic field strength. However, our design allows for simple interchange of magnets with different properties to easily modify the magnetic field.

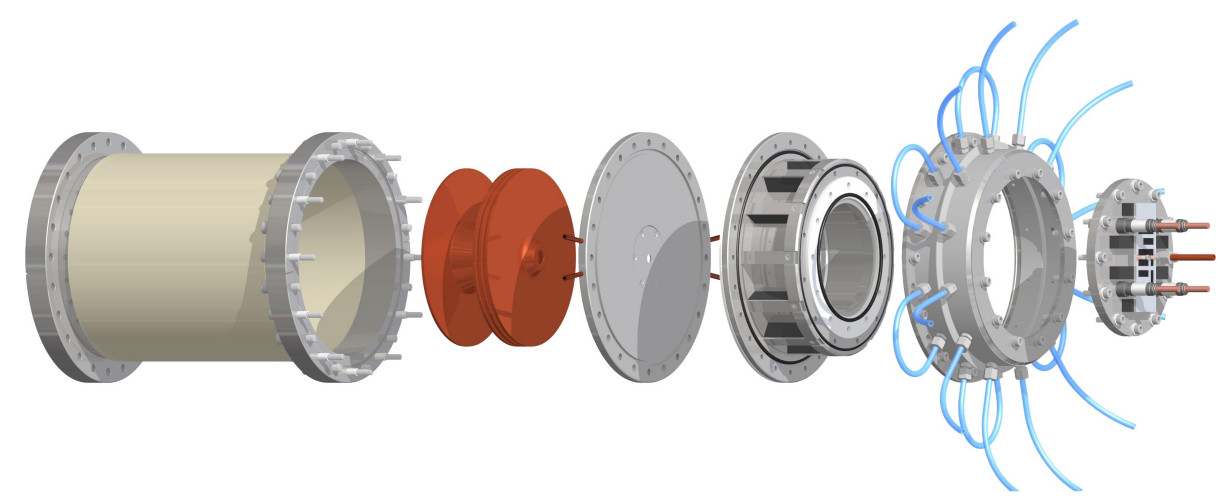

Figure 3: Components of MIST-1. From right to left: back plate, cooling water jacket, plasma chamber, front plate, extraction system, $30 \mathrm{kV}$ insulator.

In 1983, A group at the Lawrence Berkeley National Laboratory (LBNL) developed an $\mathrm{H}_{2}^{+}$ multicusp ion source and was able to reach a current density of $50 \mathrm{~mA} / \mathrm{cm}^{2}$ with an $\mathrm{H}_{2}^{+}$fraction of $80 \%$ [16]. The development strategy that we took when designing MIST-1 was to attempt to use a similar design to this source while providing more flexibility for $\mathrm{H}_{2}^{+}$optimization. The flexibility arises from several innovations: we have embedded a cooling water loop into the front plate to reduce the heat build up on the extraction aperture; we have electrically isolated the front plate, from the plasma chamber, from the back plate, which allows each component to individually biased up to $\pm 200 \mathrm{~V}$; and finally, the ion source has been designed to be modular such that each component is easily accessible for quick modifications, as shown in Fig. 3. The modularity allows us to control the length of the plasma volume by swapping out back plates with different magnet configurations, or changing the strength of the external $\mathrm{Sm}_{2} \mathrm{Co}_{17}$ magnets around the plasma chamber. Further, MIST-1 was designed in such a way that it will enable us to investigate heating the plasma using a hot filament or an external antenna and $2.45 \mathrm{GHz}$ microwaves. Testing the ion source is scheduled for early 2017.

\section{Axial injection into a cyclotron using a RFQ}

A RFQ is a compact linear accelerator that is able to focus, bunch, and accelerate a continuous beam of ions while preserving the emittance. Further, since RFQs can only efficiently transmit a single charge to mass ratio, we can use it to separate the unwanted ion species $\left(\mathrm{H}^{+}\right.$and $\left.\mathrm{H}_{3}^{+}\right)$from our $\mathrm{H}_{2}^{+}$beam. By replacing the analyzing magnets, focusing solenoids, and two-gap buncher of our conventional low energy beam transport system (LEBT) with a RFQ, we can dramatically reduce the size of our front-end while also increasing the combined capture efficiency in the cyclotron to $>40 \%$. Also, since the RFQ accelerates the beam, the ion source can be placed on a much lower potential, thus simplifying the design and installation of IsoDAR's front-end. 


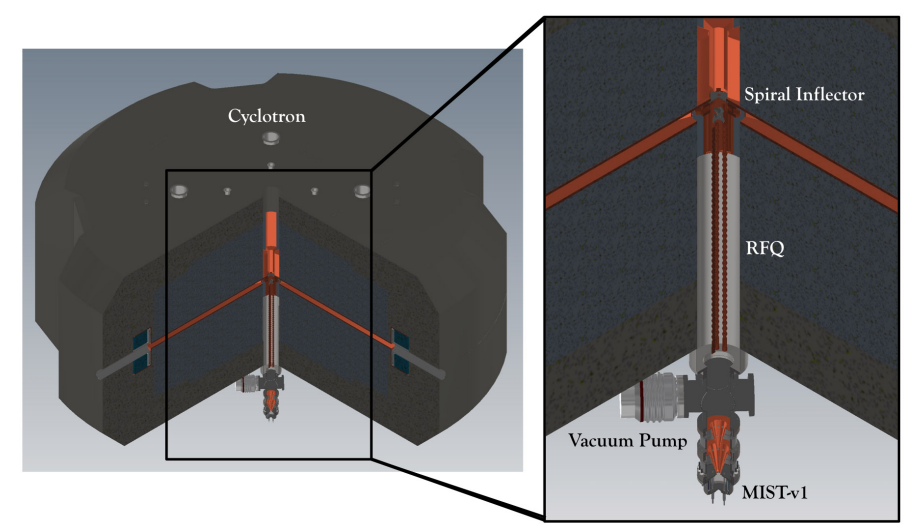

Figure 4: Rendering of a potential coupling method between the ion source, RFQ, and cyclotron. The RFQ is embedded into the yoke of the cyclotron and is positioned very close to the spiral inflector.

The original idea to use a RFQ in the axial injection system of a cyclotron was first proposed in 1981, at Los Alamos National Laboratory [17], but it has yet to be realized. In collaboration with one of the original authors of this proposal, a preliminary feasibility study was conducted using the software package PARMTEQ [18]. The simulation results [19, 20] for a $33.2 \mathrm{MHz}, 1.02 \mathrm{~m}$ long RFQ indicate that we can accelerate $10 \mathrm{~mA}$ of $\mathrm{H}_{2}^{+}$to $80 \mathrm{keV}$ through the RFQ with a transmission efficiency of $99 \%$ and full ion species separation. The simulations also indicate that the RFQ must be inserted into the yoke of the cyclotron and that we may need a supplementary focussing element before the spiral inflector in order to avoid substantial loss of beam due to divergence. A conceptual image of this design is shown in Fig. 4.

\section{Conclusion}

IsoDAR is a decisive sterile neutrino experiment that pushes the frontier of high-intensity cyclotrons and ion sources. In order to achieve the desired neutrino flux for IsoDAR, the front-end and cyclotron should be able to deliver approximately $10 \mathrm{~mA}$ of protons to the berylium target. To increase the $\mathrm{H}_{2}^{+}$current extracted from the cyclotron, two new approaches are being pursued. Frist, we have designed a new high-intensity multicusp ion source, MIST-1, that offers flexibility to optimize the $\mathrm{H}_{2}^{+}$output. It is currently being commissioned at MIT and we expect to have preliminary results in early 2017. The second approach is to use a RFQ in place of the low-energy beam transport system. This is due to the RFQ's unique ability to bunch, focus, and accelerate a continuous beam of ions while reducing the size of IsoDAR's front-end and lowering the requirements from the ion source. Our preliminary simulations indicate that the RFQ is a very efficient method to accelerate and bunch the beam, however the beam becomes highly divergent at the exit of the RFQ. New simulations including a supplementary focusing element are currently underway and we expect to have a new design results in mid-2017. 


\section{References}

[1] J. N. Abdurashitov, et al. Measurement of the solar neutrino capture rate with gallium metal. Results for the 2002-2007 data-taking period, Phys. Rev. C 80.1, 015807 (2009).

[2] Kaether, Florian, et al. Reanalysis of the GALLEX solar neutrino flux and source experiments, Phys. Letters B 685.1, 47-54 (2010).

[3] G. Mention, et al. Reactor antineutrino anomaly, Phys. Rev. D 83.7, 073006 (2011).

[4] A. Aguilar, et al. Evidence for neutrino oscillations from the observation of $\bar{v}_{e}$ appearance in a $\bar{v}_{\mu}$ beam, Phys. Rev. D 64.11, 112007 (2001).

[5] A.A. Aguilar-Arevalo, et al. Unexplained excess of electronlike events from a 1-GeV neutrino beam, Phys. Rev. Lett. 102, 101802 (2009).

[6] J. M. Conrad, et al. Precision $\bar{v}_{e}$ e-electron scattering measurements with IsoDAR to search for new physics, Phys. Rev. D 89.7, 072010 (2014).

[7] B. E. Berger, et al. The KamLAND full-volume calibration system, JINST 4.04, P04017 (2009).

[8] T. Adam, et al. JUNO conceptual design report, arXiv preprint arXiv $\mathbf{1 5 0 8 . 0 7 1 6 6}$ (2015).

[9] C. Giunti, et al. Pragmatic view of short-baseline neutrino oscillations, Phys. Rev. D 88.7, 073008 (2013).

[10] J. M. Conrad, et al. Sterile neutrino fits to short - baseline neutrino oscillation measurements, Advances in High Energy Physics (2013).

[11] J. M. Conrad, and M. H. Shaevitz, Electron antineutrino disappearance at KamLAND and JUNO as decisive tests of the short baseline $\bar{v}_{\mu} \rightarrow \bar{v}_{e}$ appearance anomaly, Phys. Rev. D 89.5, 057301 (2014).

[12] J. Alonso, et al. The IsoDAR high intensity $\mathrm{H}_{2}^{+}$transport and injection tests, JINST 10.10, T10003 (2015).

[13] G. Castro, et al. A new $\mathrm{H}_{2}^{+}$source: conceptual study and experimental test of an upgraded version of the VIS - Versatile Ion Source, Rev. Sci. Inst. 87.8, 083303 (2016).

[14] I.N.D.S.A.D. Unit, "Aladdin." https://www-amdis.iaea.org/ALADDIN/, Accessed: September 2015.

[15] S. Axani, et al. A high intensity $\mathrm{H}_{2}^{+}$multicusp ion source for the isotope decay-at-rest experiment, IsoDAR, Rev. Sci. Inst. 87.2, $02 \mathrm{~B} 704$ (2016).

[16] K. W. Ehlers and K. N. Leung, High - concentration $\mathrm{H}_{2}^{+}$or $D_{2}^{+}$ion source, Rev. Sci. Inst. 54.6, 677-680 (1983).

[17] R.W. Hamm, D. A. Swenson, and T. P. Wangler, Use of the radio-frequency quadrupole structure as a cyclotron axial buncher system, No. CONF-810944-11 (1981).

[18] K.R. Crandall, and T.P. Wangler. PARMTEQ - A beam-dynamics code fo the RFQ linear accelerator, Linear Accelerator and Beam Optics Codes., Vol. 177. No. 1. AIP Publishing (1988).

[19] D. Winklehner, R. Hamm, J. Alonso, J. Conrad, and S. Axani, Preliminary design of a RFQ direct injection scheme for the IsoDAR high intensity $\mathrm{H}_{2}^{+}$cyclotron, Rev. Sci. Inst. 87(2), $02 \mathrm{~B} 929$ (2016).

[20] D. Winklehner, R. Hamm, J. Alonso, J. Conrad, An RFQ direct injection scheme for the IsoDAR high intensity H+2 cyclotron, Proceedings of the 6th International Particle Accelerator Conference IPAC (2015). 